\title{
Decompression of the Suprascapular Nerve at the Superior Scapular Notch Under Combined Arthroscopic and Ultrasound (US) Guidance: US- Arthroscopy
}

Clément Prénaud ( $\nabla$ clementprenaud@gmail.com )

Assistance Publique - Hôpitaux de Paris

Jeanne LOUBEYRE

Assistance Publique - Hôpitaux de Paris

Marc SOUBEYRAND

Clinique Saint Jean Ermitage

\section{Research Article}

Keywords: Suprascapular, nerve, decompression, arthroscopic, ultrasound

Posted Date: May 17th, 2021

DOI: https://doi.org/10.21203/rs.3.rs-366928/v2

License: (c) (i) This work is licensed under a Creative Commons Attribution 4.0 International License.

Read Full License 


\section{Abstract}

Background: Decompression of the suprascapular nerve (SSNe) at the superior scapular notch (SSNo) is usually performed with an arthroscopic procedure. This technique is well described but locating the nerve is complex because it is deeply buried and surrounded by soft tissue. We propose to combine ultrasound and arthroscopy (US-arthroscopy) to facilitate nerve localization, exposure and release. The main objective of this study was to assess the feasibility of this technique.

Methods: This is an experimental, cadaveric study, carried out on shoulders. The first step of our technique is to locate the SSNo using an ultrasound scanner. Then an arthroscope is introduced under ultrasound control to the SSNo. A second portal is then created to dissect the pedicle and perform the ligament release.

Results: Ultrasound identification of the SSNo, endoscopic dissection and decompression of the nerve were achieved in $100 \%$ of cases. Ultrasound identification of the SSNo took an average of min (+/-4) while dissection and endoscopic release time took an average of $\min (+/-5)$.

Conclusion: Ultrasound is an extremely powerful tool for non-invasive localization of nerves through soft tissues, but it is limited by the fact that tissue visualization is limited to the ultrasound slice plane, which is two-82 dimensional. On the other hand, arthroscopy (extra-articular) allows three-dimensional control of the surgical steps performed, but the locating of the nerve involves significant tissue detachment and a risk of damaging the nerve with the dissection. The combination of the two (US-arthroscopy) offers the possibility of combining the advantages of both techniques.

\section{Full Text}

This preprint is available for download as a PDF.

\section{Figures}




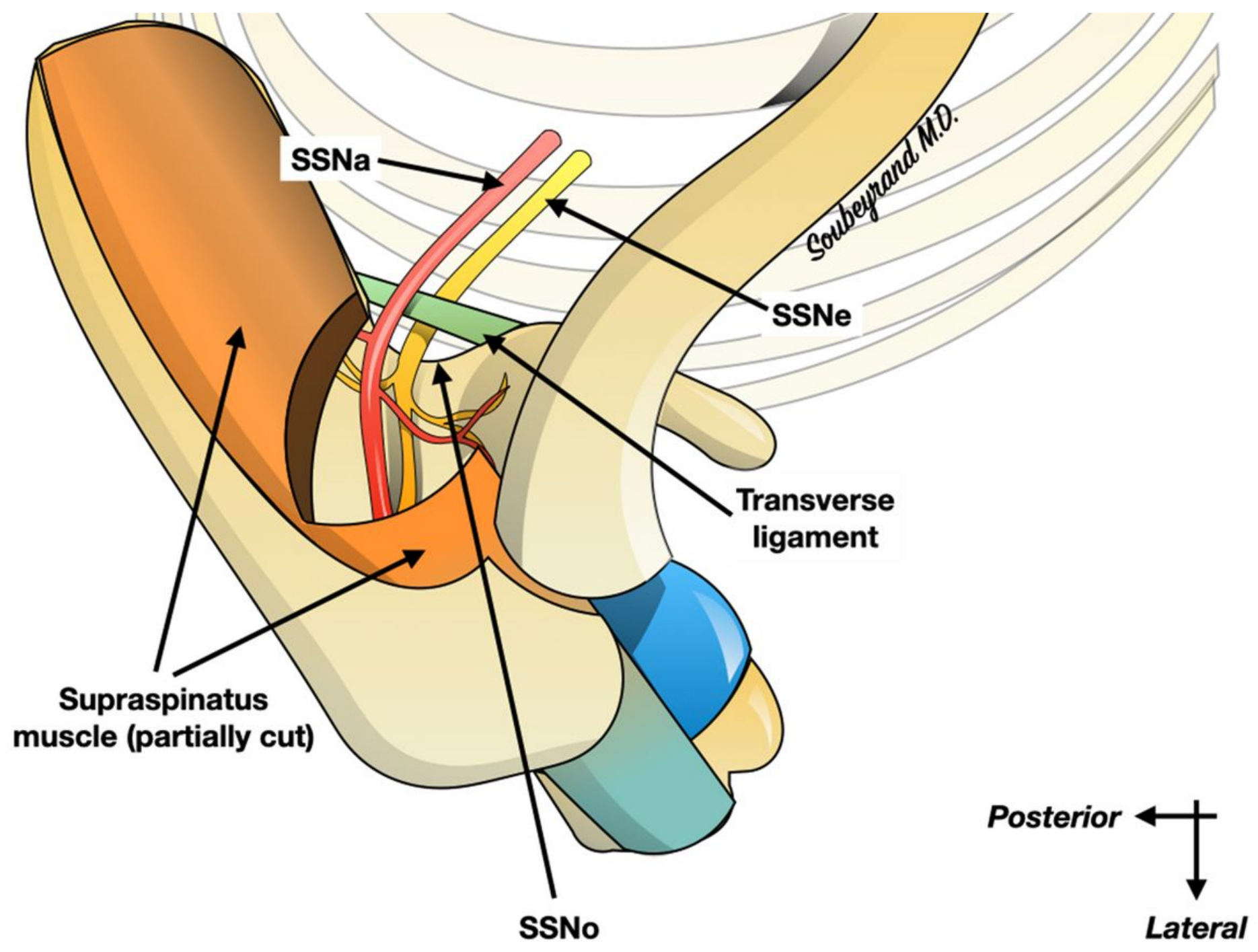

Figure 1

Anatomy of the suprascapular nerve ( $\mathrm{SSNe}$ ) passing through the suprascapular notch (SSNo), under the transverse ligament, accompanied by the artery (SSA). The SSNe supplies the supraspinatus muscle as well as the infraspinatus muscle (not shown on the illustration). 


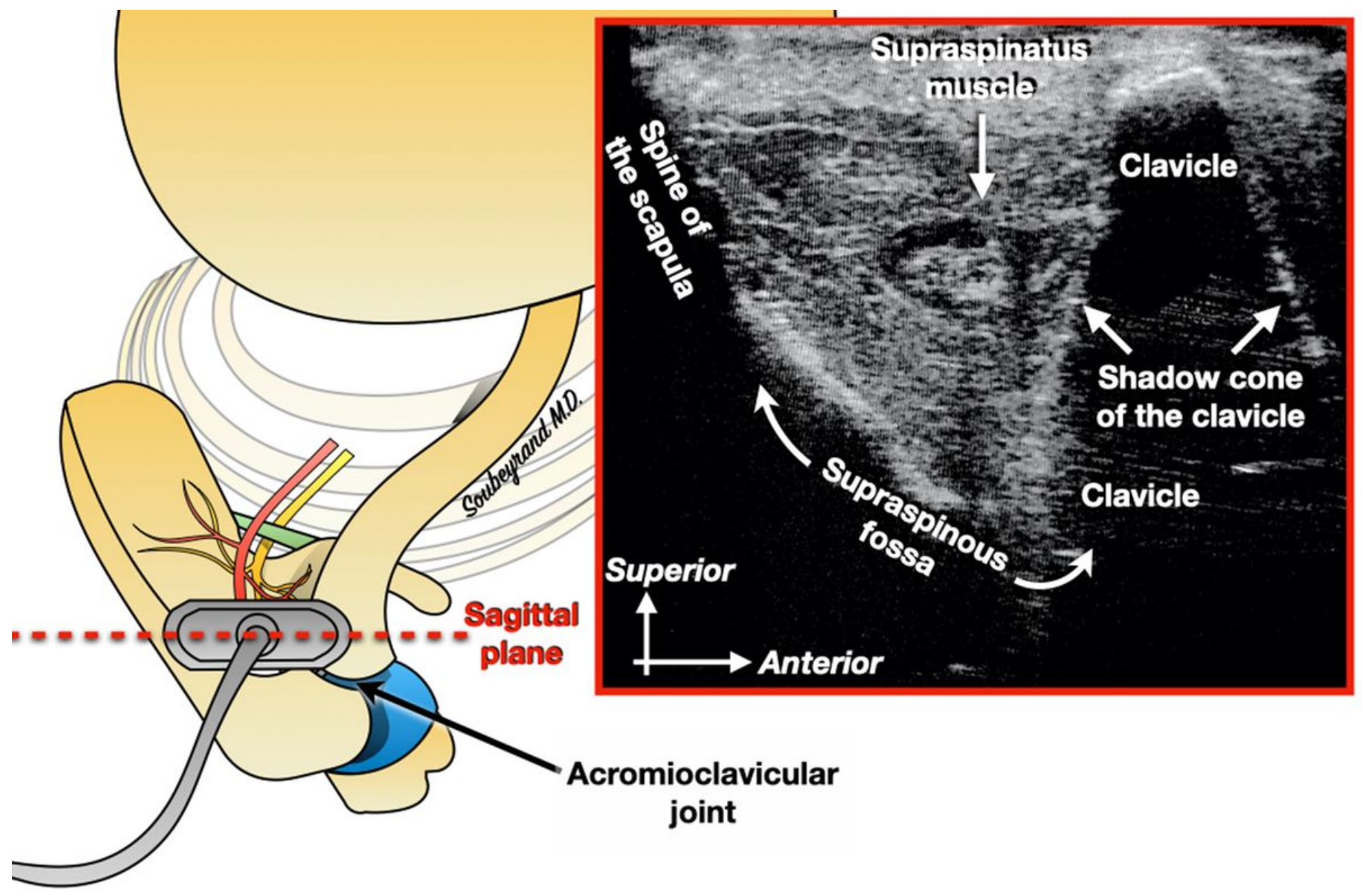

Figure 2

The ultrasound probe is positioned in the sagittal plane, just behind the acromioclavicular joint. The supraspinous fossa can thus be identified. 


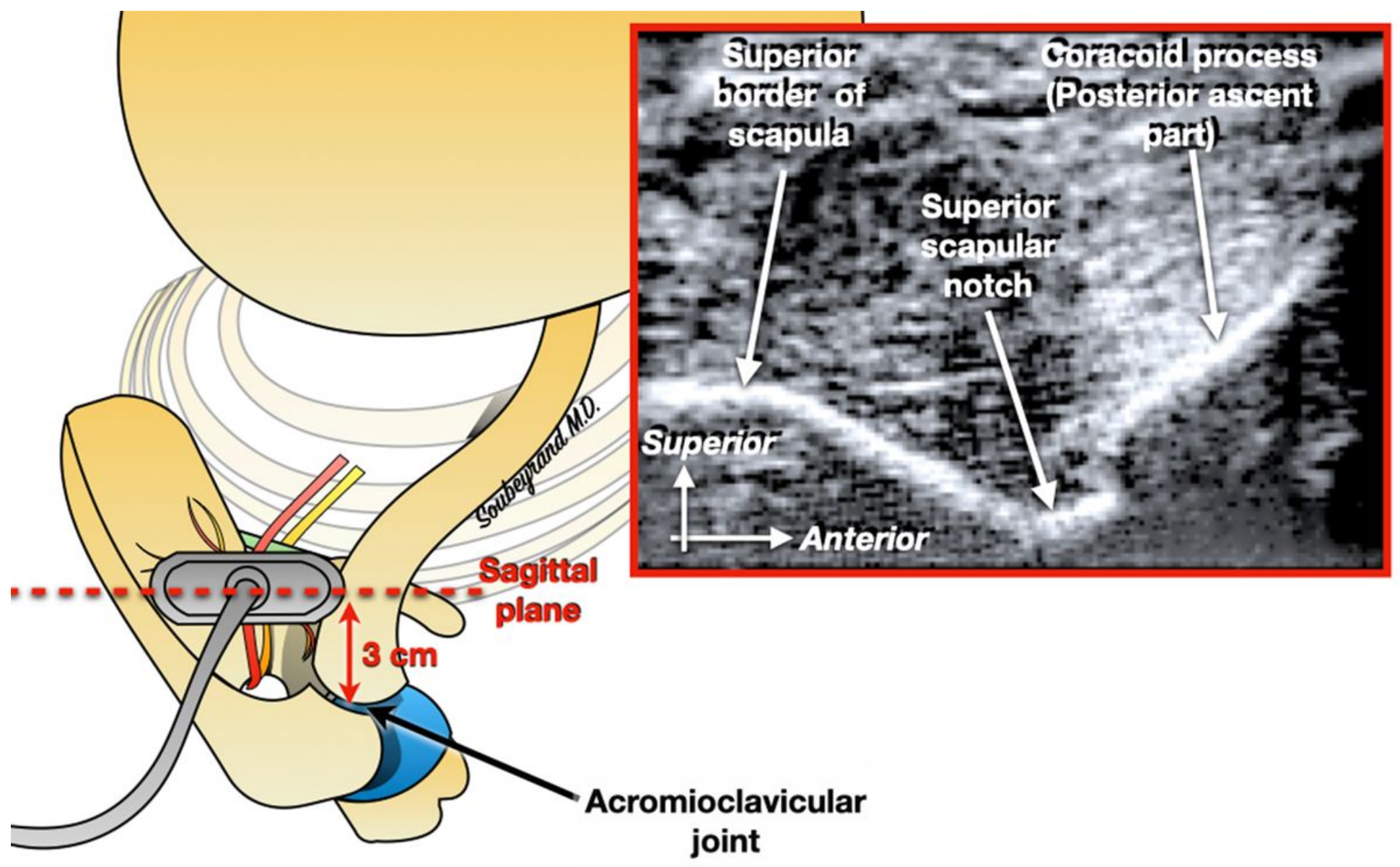

Figure 3

then the probe is shifted about $\mathrm{cm}$ medially to the acromioclavicular joint. This makes it possible to locate the superior scapular notch. 


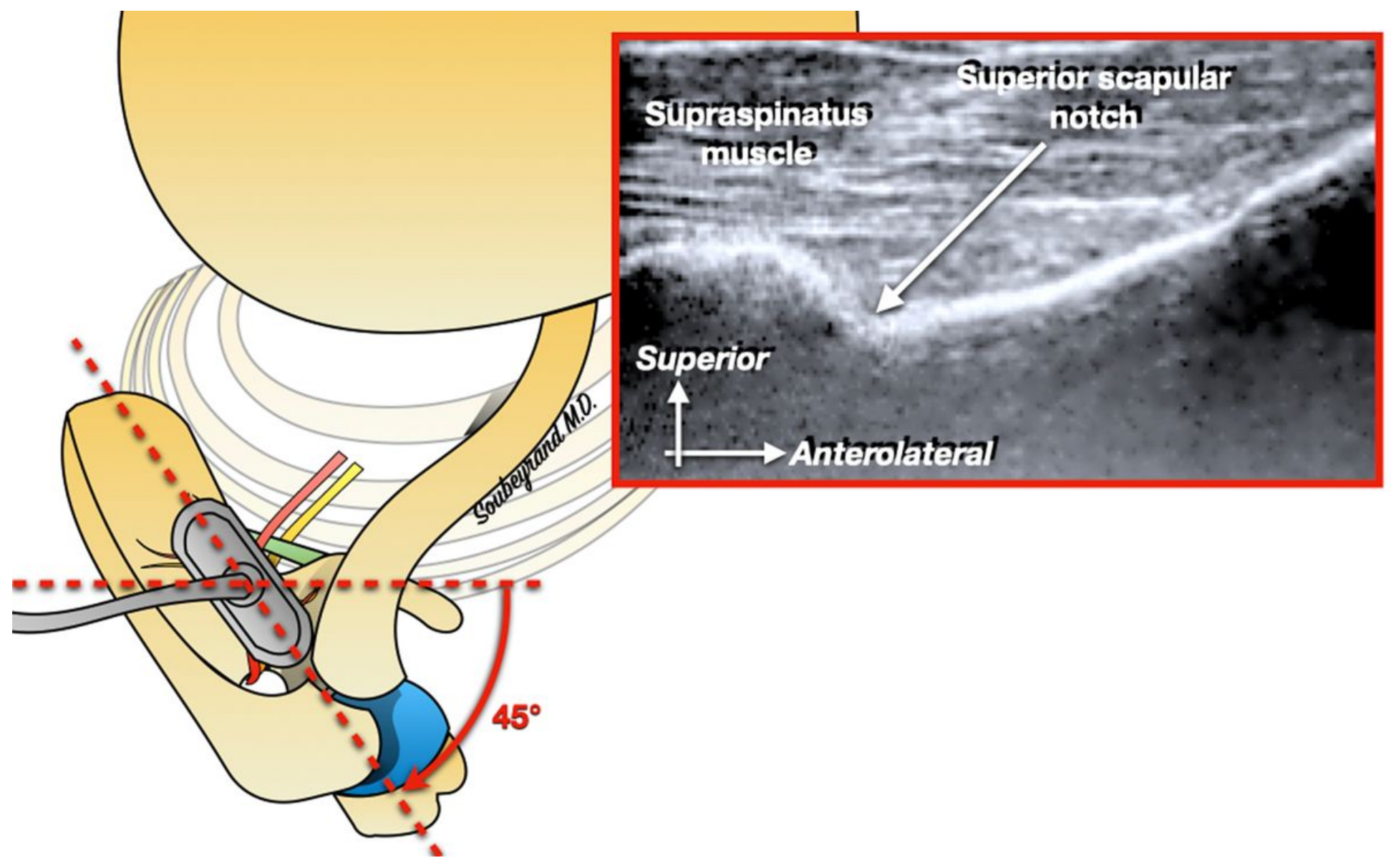

Figure 4

The probe is then rotated about $45^{\circ}$, parallel to the body of the scapula. 


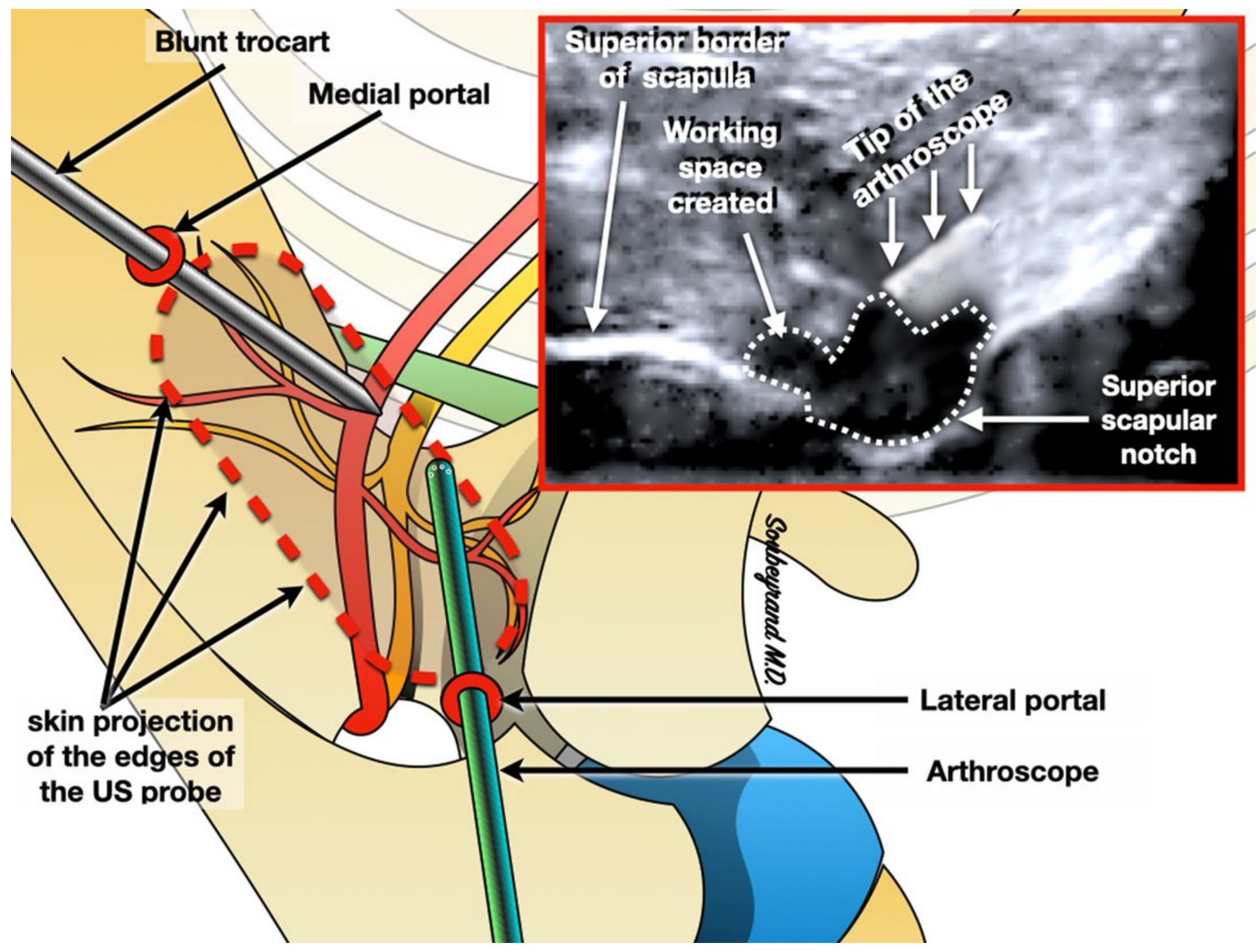

Figure 5

Two arthroscopic portals are created on either side of the probe (medial and lateral) in order to introduce the arthroscope and a blunt trocar. The trocar is used to create a working space around the notch: its tip is used to detach the soft tissue. The workspace gradually appears as a hypoechoic area. The arthroscope is then introduced under ultrasound guidance into the working area. 


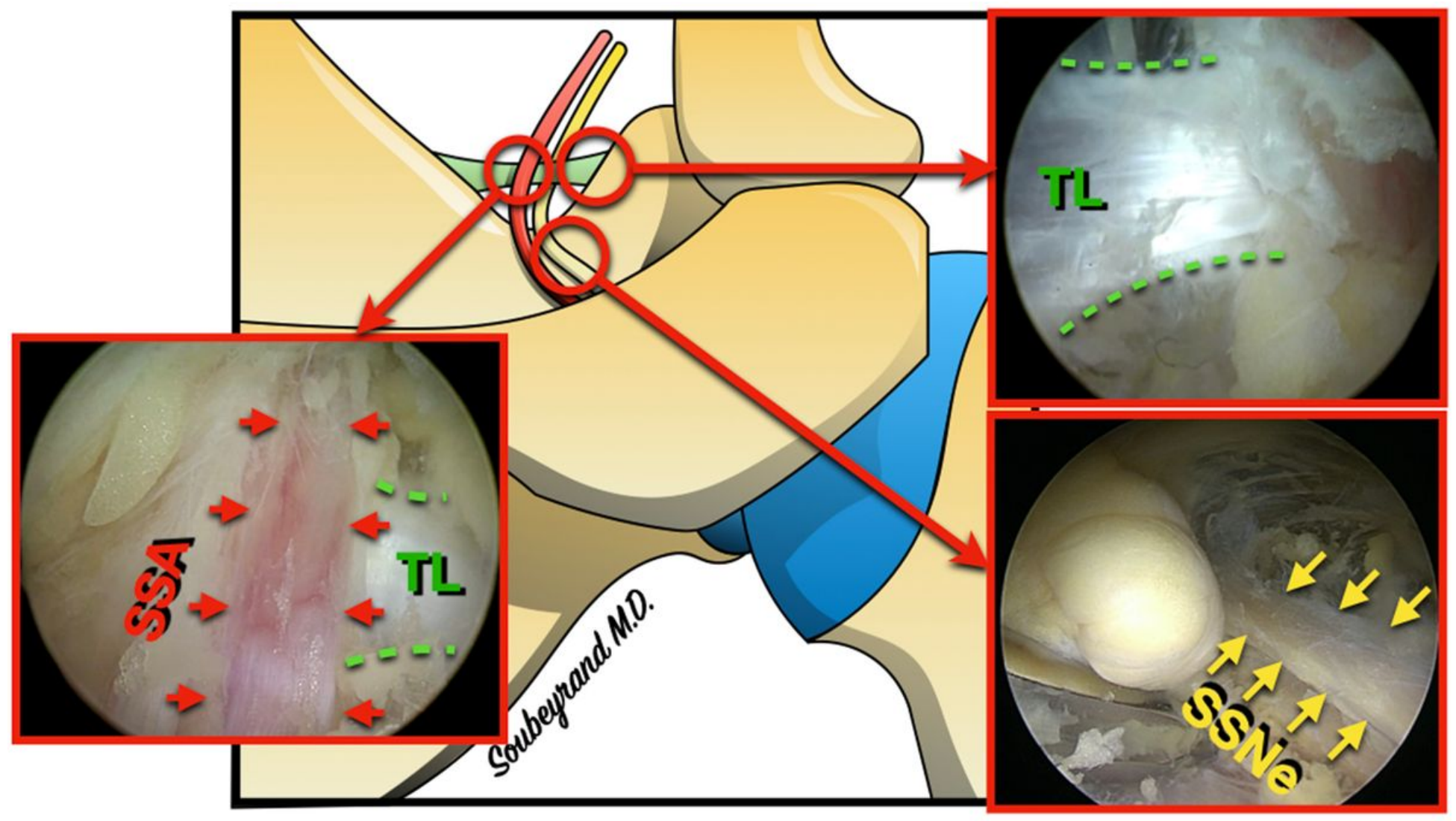

Figure 6

Arthroscopic aspect of the suprascapular notch (SSNo) with the suprascapular nerve (SSNe), suprascapular artery (SSA) and transverse ligament (TL). 


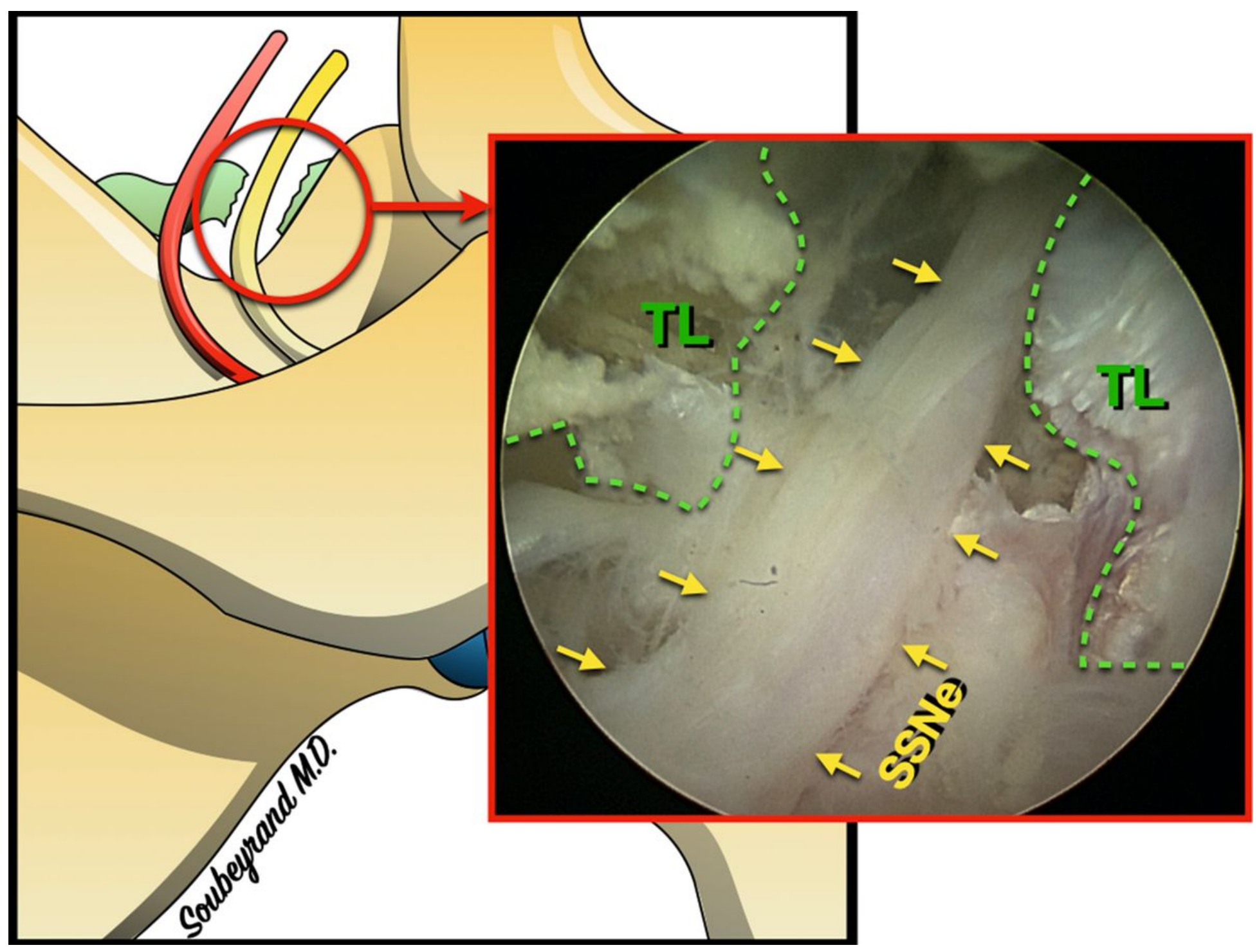

Figure 7

Arthroscopic appearance after section of the transverse ligament (TL): the suprascapular nerve (SSNe) appears completely released. 